Cite as: F. Stilp et al., Science

10.1126/science.abe2600 (2021).

\title{
Very weak bonds to artificial atoms formed by quantum corrals
}

\author{
Fabian Stilp', Andreas Bereczuk², Julian Berwanger', Nadine Mundigl1, Klaus Richter ${ }^{2}$, Franz J. Giessibl ${ }^{*}$ \\ ${ }^{1}$ Institute of Experimental and Applied Physics, Department of Physics, University of Regensburg, 93040 Regensburg, Germany. ${ }^{2}$ Institute of Theoretical Physics, \\ Department of Physics, University of Regensburg, 93040 Regensburg, Germany. \\ ${ }^{*}$ Corresponding author. Email: franz.giessibl@ur.de
}

We explored the bonding properties of the quantum corral (a circle of 48 iron atoms placed on a copper surface) reported by Crommie, Lutz and Eigler in 1993, along with variants, as an artificial atom using an atomic force microscope (AFM). The original corral geometry confines 102 electrons to 28 discrete energy states, and we find that these states can form a bond to the front atom of the AFM with an energy of about 5 millielectron volts. The measured forces are about 1/1000 of typical forces in atomically resolved AFM. The confined electrons showed covalent attraction to metal tips and Pauli repulsion to CO-terminated tips. The repulsion at close distance was evident from the response of corral states created by deliberately placing single iron atoms inside the corral. The forces scaled appropriately with a 24-atom corral.

Artificial atoms are structures that confine a fixed number of electrons in a small space such that these electrons show discrete energy levels (1). The original quantum corral, a circular arrangement of $48 \mathrm{Fe}$ atoms with a radius of $r_{\mathrm{C}}=7.13 \mathrm{~nm}(2)$ on a $\mathrm{Cu}$ (111) surface, was created by atomic manipulation (3) with a scanning tunneling microscope (STM). We studied the chemical bonding of this artificial atom (and structures with extra Fe atoms and a smaller 24-atom corral) with single atom metal tips and CO-terminated tips of an atomic force microscope (AFM) (4). We observed an attractive interaction between the corral states and the front atom of the metal tip for core-core distances of the front atom of the tip and the plane of the surface $\mathrm{Cu}$ atoms from 620 to 440 p.m. (6.2 to 4.4 $\AA$ ). The measured bonding energy between the single atom metal tip and the artificial atom of $5 \mathrm{meV}$ is only a fraction of the van der Waals (vdW) bonding energy between two xenon atoms that amounts to $35 \mathrm{meV}(5)$. The $\mathrm{CO}$ terminated tip can approach the surface down to a distance of 410 p.m., it interacts repulsively with the corral states. Because of the weak interaction, a reasonable signal-to-noise ratio is only obtainable up to a distance of about 490 p.m.

The artificial atom manifested by the corral states does not contain a fixed number of electrons as it is connected to an electron reservoir from the $\mathrm{Cu}$ substrate. We show below that the corral should contain $\approx 102$ electrons. The $\psi_{n=5, l=0}$ state, where $n$ denotes the radial zeroes of the wavefunction and $l$ is the angular momentum, has a calculated energy that is exactly at the Fermi level $E_{\mathrm{F}}$ and as the levels are broadened, this state is expected to be half-filled. Thus, the expected electron occupation is 101 electrons if the energy of the $\psi_{5,0}$ state exactly matches $E_{\mathrm{F}}$, but the $\pm 10 \mathrm{meV}$ uncertainty in that energy can lead to variations. In addition, attractive interaction with the AFM tip will shift the energy of this state to lower values, causing increased filling, and repulsive interaction will cause the opposite effect. Experimentally, the attractive interaction with a metal tip led to an occupation of up to 0.95 electrons for each of the two spin states in the $\psi_{5,0}$ state, whereas repulsive interaction with a $\mathrm{CO}$ terminated tip reduced it to 0.65 , leading to a total occupation between 101.9 and 101.3 electrons.

A scanning tunneling microscopy (STM) image of the 48atom quantum corral (2) was recorded with a CO-terminated metal tip [Fig. 1A; see section 1 in (6) for experimental details] resolved both the $\mathrm{Cu}$ surface atoms as well as the confined-electron eigenstates [see section 2 in (6) for a comparison to the data obtained with a metal tip]. The two-dimensional (2D) nature of the quantum corral originated from the surface states that exist on closely packed noble metal surfaces such as $\mathrm{Cu}(111)(7)$. Figure 1B shows the overall energy scale, where $E_{0}$ is below the vacuum level by the work function $=4.94 \mathrm{eV}(8)$ plus the occupied range of the surface band given by $440 \pm 10 \mathrm{meV}(9)$. The unperturbed surface state on $\mathrm{Cu}(111)$ can be described by a 2D quasi-free electron gas with a quadratic energy dispersion of $E^{\prime}(k)=\hbar^{2} \boldsymbol{k}^{2} /\left(2 m^{*}\right)$, where $\hbar$ is Planck's constant divided by $2 \pi$ and the effective mass $m^{*}$ is $0.38 \pm 0.02$ times the electron mass (9) (Fig. 1C); the wave vector $\boldsymbol{k}$ is parallel to the surface, with a Fermi wave vector of $k_{\mathrm{F}}$ $=2 \pi / \lambda_{\mathrm{F}} \approx 2.1 \mathrm{~nm}^{-1}$ and $\lambda_{\mathrm{F}} \approx 3 \mathrm{~nm}(10)$.

The Fe adatoms repulsively scatter the surface state, and the iron ring forces the modified surface states into a potential energy landscape that can be reasonably modeled by a disc of radius $r_{\mathrm{C}}$ with hard-wall confinement $(2,11-13)$. The solutions of the Schrödinger equation for the hard-wall model are

$$
\psi_{n, l}(r, \phi, z)=C_{n, l} \cdot J_{l}\left(\rho_{0 n, l} \cdot r / r_{\mathrm{C}}\right) \cdot e^{i l \phi} \cdot e^{-z /\left(2 \lambda_{B}\right)}
$$

in the following referred to as Bessel-type eigenstates. Each state with angular momentum $l \cdot \hbar$ in $z$ direction, where $l$ can be negative and positive, can be occupied by two electrons 
(spin up/down). The constant $C_{\mathrm{n}, \mathrm{l}}$ is a normalization constant, $J_{1}(\rho)$ is the Bessel function of the first kind of order $l$ with $n$th zero $\rho_{0 \mathrm{n}, 1}$ [figs. $\mathrm{S} 3$ to $\mathrm{S} 5$ and table $\mathrm{S} 1$ in section 3 in (6)], $r$ is the radial distance from the center of the corral, $\phi$ is the azimuthal angle and $\lambda_{\mathrm{B}}=42.1 \mathrm{pm}$ is the decay length of the electron density described by the Bessel-type eigenstates into the vacuum for all $n, l$.

The discrete energy spectrum of the Bessel-type eigenstates (Fig. 1D) showed energy level broadening caused by scattering and coupling to the bulk substrate $(2,11)$. Five of these states were visible by STM at low bias as their energy eigenvalues were near $E_{\mathrm{F}} ; \psi_{5,0}$ was exactly at the Fermi energy $E_{\mathrm{F}}$ and $\psi_{4, \pm 2}, \psi_{2, \pm 7}$ were 8 and $7 \mathrm{meV}$ below $E_{\mathrm{F}}$, respectively. Because of the $\pm 10 \mathrm{meV}$ uncertainty in the occupied range of the surface band and the uncertainty of the effective mass, the occupation of each spin state in $\psi_{5,0}$ could be anywhere between 0 and 1 . Because of the finite energy width of the $\psi_{5,0}$ state, its occupation would be 0.5 if the occupied range of the surface band was exactly $440 \mathrm{meV}$ and $m^{*}=0.38 m_{\mathrm{e}}$. The occupation of the $\psi_{5,0}$ state was modified by the tip-sample interaction.

Figure $1 \mathrm{E}$ shows the surface charge density $\sigma_{\mathrm{Fermi}}$ of these five states when fully occupied by a total of ten electrons, the dashed line is $\sigma_{\mathrm{Fermi}}$ with empty $\psi_{5,0}$ states. The experimental STM data of Fig. 1A, fig. S1A in (6), and fig. 2B in (2) resemble $\sigma_{\mathrm{Fermi}}$. The remaining 92 electrons have an energy far below $E_{\text {F. }}$. Thus, a total of 102 electrons occupy the corral if the $\psi_{5,0}$ states are filled, yielding an average surface charge density of $\sigma_{\mathrm{av}}=102 \mathrm{e} /\left(\pi r_{\mathrm{C}}{ }^{2}\right)=0.639 \mathrm{e} / \mathrm{nm}^{2}$. In the profile of the calculated total surface charge density $\sigma_{\text {total }}$ (Fig. $1 F$ ), the dashed line is $\sigma_{\text {total }}$ without the contribution of $\psi_{5,0}$ states. The surface charge density of the radially rippled disc-shaped cloud of electrons in the corral was very dilute compared to natural atoms. For example, $\mathrm{Cu}$ has an atomic radius of $127.5 \mathrm{pm}$ and an average surface charge density for its 11 valence electrons of $\approx 215 \mathrm{e} / \mathrm{nm}^{2}$ when projected on a plane (14), or $\sim 340$ times the surface charge density of the corral.

To investigate the bonding properties of the quantum corral, we used frequency-modulation AFM (15), which can achieve atomic resolution and deliver low-noise force spectroscopy $(16,17)$. We use two types of AFM tips, single-atom metal tips that often form strong bonds with conventional atoms (18), and CO-terminated metal tips (19) that are usually chemically inert (20) and interact mainly through Pauli repulsion $(21,22)$. Electrostatic interactions of the corrugated electron states caused only negligible interaction forces [see section 4 in (6)].

The interaction of the surface and corral states with a single-atom metal AFM tip is shown in Fig. 2, A to E, and a COterminated AFM tip in Fig. 2, F to J. The scan size was confined to the central area of the ring to prevent the tip from colliding with the Fe corral atoms. A constant height mode at close distance was required because the vdW force between tip and sample was 10 000 times larger than the bonding force between quantum corral and tip, and a very small change in height would induce a large change in vdW forces. Careful checks ruled out cross talk between current and force [see section 5 in (6)].

The raw data of the frequency shift recorded with a singleatom metal tip (Fig. 2A) was low-pass filtered to produce Fig. 2B. Both attractive and repulsive interactions apparently scaled well with the total charge density [see section 6 in (6)], so in Fig. 2C, we compared a frequency shift profile (left vertical axis) taken at constant height to the reverse total charge density of the corral states (right vertical axis, reversed) from Fig. 1F. The comparison of frequency shifts to surface charge densities is motivated by prior experiments on the interaction of metal tips with adatoms (18) and CO tips with organic molecules (20) as well as calculations on Pauli repulsion of CO tips $(21,22)$ where the force and thus the frequency shift was approximately proportional to the charge density. The decrease of the ripples' contrast with distance on an $(x, z)$ plane can be seen in Fig. 2D, and Fig. 2E displays the exponential fading with distance of frequency shift and force (fit line in Fig. 2E). The experimental decay length of $\lambda_{\text {met }}=55.7$ $\mathrm{pm}$ is near the calculated decay length of the charge density of the Bessel-type eigenstates $\lambda_{\mathrm{B}}=42.1 \mathrm{pm}$ of Eq. 1 . The acquisition of this low-noise data requires an averaging technique that is explained in section 7 in (6).

The data for a CO terminated tip (Fig. 2, F to J) had two marked differences compared to the metal tip data. First, as expected from previous studies $(23,24)$, we observed an attractive (dark) image of the $\mathrm{Cu}(111)$ surface atoms spaced by $255 \mathrm{pm}$ in Fig. 2F. Second, the faint contrast of the ripples caused by the Bessel-type eigenstates was repulsive and only $\sim 10 \%$ of the magnitude for the metal tip of Fig. $2 \mathrm{~A}$ at the same height $z$. The low-pass filtered version (Fig. 2G) of Fig. 2F showed the ripples more clearly but suppressed the atomic surface lattice.

The red trace in Fig. $2 \mathrm{H}$ shows an experimental frequency shift profile at constant height, again compared to a profile of the total charge density of the electrons in the corral. The fit between the experimental data and the total charge density was reasonable, except for the central peak that was much higher in the total charge density compared to the experimental data. A nearly perfect fit was obtained by setting the occupancy of each of the two spin states in $\psi_{5,0}$ that was closest to $E_{\mathrm{F}}$ to 0.7 [section 8 in (6); the reasoning for that occupancy discussed below]. Figure 2I showed the contrast evolution as a function of distance $z$, where the fit line in Fig. $2 \mathrm{~J}$ resulted in a decay length of $\lambda_{\mathrm{CO}}=50.4$ p.m., again near the calculated vertical decay length of the charge density from Eq. 1 of $\lambda_{\mathrm{B}}=42.1 \mathrm{pm}$ and slightly less than for the metal tip with $\lambda_{\text {metal }}=55.7$ p.m. 
We conclude for the analysis of the data in Fig. 2 that the corral artificial atom interacted with the AFM probe tips in a similar way as natural atoms or molecules. For the metal tip, we observed covalent bonding, that is, an attractive interaction with the $2 \mathrm{D}$ corral atom. For a $\mathrm{CO}$ terminated tip, we observed Pauli repulsion between tip and the 2D corral atom (20).

Back-action in the measurement of a quantum state is a basic principle of quantum mechanics, and the proximity of the AFM tip will alter the corral quantum mechanical states, just as surface atoms interact via surface states (25-27). One effect of observation is the alteration of energy eigenvalues $E_{\mathrm{n}, \mathrm{l}}$ under the influence of an external potential $V_{\mathrm{ts}}$ where the shifted energy $E_{n, 1}^{*}$ is approximated by perturbation theory:

$$
E_{n, l}^{*}=E_{n, l}+\left\langle\psi_{n, l}\left|V_{\mathrm{ts}}\right| \psi_{n, l}\right\rangle
$$

Of all the occupied states in the quantum corral, the $\psi_{5,0}$ state was most sensitive to occupation changes induced by the tip-sample interaction as its energy eigenvalue is directly at $E_{\mathrm{F}}$ [see table S2 in (6) and Fig. 1D], and the finite width of the eigenstate energies must be considered. The quantum corral was coupled strongly to the $\mathrm{Cu}(111)$ substrate (11-13), which broadens the energy levels, as found experimentally in (2). According to the hard wall model, the energy of the $\psi_{5,0}$ state is exactly $440 \mathrm{meV}$ [table S2 in (6)]. Given that the occupied range of the surface state band has some uncertainty $[440 \pm 10 \mathrm{meV}(9)]$, the $\psi_{5,0}$ state should be about half-filled if the AFM tip is far away.

A repulsive interaction with the tip, where $\left\langle\psi_{5,0}\left|V_{\mathrm{ts}}\right| \psi_{5,0}\right\rangle$ $>0$, reduces the filling of the $\psi_{5,0}$ state, and an attractive interaction with its negative matrix element increases its filling [see fig. S11 in (6)]. AFM can measure $V_{\text {ts }}$ by integrating the force from a large distance to a close distance. All occupied states contribute to the force, but only the five occupied states with $l=0$ are present in the center of the corral, and the weight of the five occupied states $\psi_{\mathrm{n}, 0}$ is proportional to the square of their respective amplitudes at $r=0$. Thus, we can estimate the energy shift for one electron in a $\psi_{5,0}$ state to be $-0.8 \mathrm{meV}$ for the metal tip and $+130 \mu \mathrm{eV}$ for the COterminated tip for the closest distance $z$ that was probed in the center of the corral. When adding up the contribution of all occupied states in the center of the corral $\psi_{\mathrm{n}, 0}$ from $\mathrm{n}=1$ to 5 as explained in section 9 in (6), we obtained a bond energy for the metal tip of $-5 \mathrm{meV}$ and an (anti-) bond energy of about $+1 \mathrm{meV}$ for the CO-terminated tip. The repulsive interaction of the $\mathrm{CO}$ terminated tip led to a lower occupation of the $\psi_{5,0}$ state than what was observed for the attractive metal tip [section 8 in (6)]. Furthermore, close inspection of Fig. 2D shows that the height ratio (HR) between the central negative peak and the peaks left and right to it decreased when increasing the distance $z$ from the minimum of $490 \mathrm{pm}$ to 570 p.m., whereas the data for the CO tip in Fig. 2I indicated that the HR rose slightly for increasing distances. The data showed that the attractive interaction of the metal tip led to a substantial increase of the population of the $\psi_{5,0}$ state but the weak repulsive interaction of the $\mathrm{CO}$ terminated tip only slightly depopulated it (6).

The perturbation caused by the tip on the corral states, based on the traces that make up Fig. 2D, is shown in Fig. 3A. The HR between the central peak and its two peaks next to it (Fig. 3B) decreased from $\sim 2.2$ at $440 \mathrm{pm}$ away to $\sim 1.2$ for 540 $\mathrm{pm}$ away for the metal tip, but for the CO tip, it increased from $\sim 0.6$ to $\sim 0.9$ over the same distance range. The HR is related to the occupation of the $\psi_{5,0}$ state (Fig. 3B), and the reduction with the $\mathrm{CO}$ tip fits to a repulsive interaction that partially depopulated this state.

Note that the blue trace in Fig. 3B rose to larger HRs for distances decreasing to 440 p.m., indicating increasing attraction that led to the loss of the front atom of the tip when approaching closer [see section 10 of (6)]. However, the transition from attraction to repulsion between the metal atom and the corral states for very close distances could be inferred from the response of the corral states when placing an $\mathrm{Fe}$ adatom at the center of the corral. Placing the adatom as a substitute for the tip directly on the $\mathrm{Cu}$ substrate at a core distance of $\approx 200 \mathrm{pm}$ to the surface plane should reduce the occupation of the $\psi_{5,0}$ state if it interacts repulsively, as shown as an STM topograph in Fig. 3C. In a constant-height AFM image of a frame with size $4.7 \mathrm{~nm}$ (Fig. 3D; see the green square in Fig. 3C), a trace through that image did not allow a clear assessment that the occupation of the $\psi_{5,0}$ state has changed as expected. However, scanning along a trace with a length of $12 \mathrm{~nm}$ (red line in Fig. 3C) covered a sufficiently large width of the corral states. In the experimental frequency shift (Fig. 3E), the green dashed line (total charge density for empty $\psi_{5,0}$ states) is lower than the black dashed curve (total charge density for a fully occupied $\psi_{5,0}$ state-note reversed scales). The match of the green dashed line to the experimental data in red suggests that the $\psi_{5,0}$ state is indeed empty [see fig. S22 in (6)].

We used STM to confirm the depletion of the $\psi_{5,0}$ state. If the two electrons that occupy the state were forced into the $\mathrm{Cu}$ substrate, the effect is larger in STM, which probes 10 versus 102. In Fig. 3C, the similar height of second and third rings of the corral states was confirmed by analyzing the traces in Fig. 3F. The red line is the experimental topographic trace, and the dashed blue and green lines show the surface charge densities at the Fermi level with a fully occupied and an empty $\psi_{5,0}$ state, respectively. The dashed green line with the empty $\psi_{5,0}$ state again showed better agreement to the experimental data in red outside the locations where the $\mathrm{Fe}$ adatoms (center and corral) contributed strongly to the current, as the tunneling channel into those states were not contained in the simulations. 
Moving the additional adatom outside of the center lifts the degeneracy of $l \neq 0$ states with $\cos (l \phi)$ and $\sin (l \phi)$ azimuthal functions. The perturbative energy shift described in Eq. 2 is only valid for non-degenerate states, so we needed to compose new linear combinations of the degenerate $\pm l$ solutions expressed in Eq. 1 that diagonalize the perturbation operator. For the $\psi_{2, \pm 7}$ states, we created two linear combinations of the angular functions $\exp ( \pm \mathrm{i} l \phi)$. The even solution $\cos (l \phi)=(\exp (\mathrm{i} l \phi)+\exp (-\mathrm{i} l \phi)) / 2$ has a maximal interaction with the extra atom at $\phi=0$ and the odd solution $\sin (l \phi)=(\exp (\mathrm{i} l \phi)-\exp (-\mathrm{i} l \phi)) / 2 \mathrm{i}$ has a minimal interaction at this angle. If the atom interacts repulsively, the energy eigenvalue of the $\cos (l \phi)$-solution will be higher than the one of the $\sin (l \phi)$-solution such that the population of the $\cos (l \phi)$-solution decreases.

Figure $3 \mathrm{G}$ shows the adatom at $x \approx 4 \mathrm{~nm}$ where the $\psi_{2, \pm 7}$ states have a local maximum. The perturbation imposed by the adatom leads to the emergence of angular ripples with a $\sin ^{2}(7 \phi)$ dependence and 14 azimuthal local minima for angles $\phi_{m}=2 \pi m / 14$ where $m$ is an integer that runs from 0 to 13. Figure $3 \mathrm{H}$ shows the more precise numerical calculation with the tight-binding based code KWANT (28), explained in detail in section 11 in (6). The tight binding simulation of Fig. $3 \mathrm{H}$ revealed subtle details not observed in modelling by Bessel type eigenstates of Eq. 1. For example, the potential energy imposed by the $48 \mathrm{Fe}$ atoms is not exactly invariant with the azimuthal angle $\phi$, but invariant with respect to rotations of multiples of $60^{\circ}$. This shows up both in experiment and calculations, as the central rings in Fig. 3, G and H, are clearly hexagonal.

We repeated these measurements for a smaller corral with a radius of $r_{\mathrm{C}}{ }^{\prime}=3.565 \mathrm{~nm}$ and 24 atoms and obtained similar bonding properties, where Fig. 4A shows an STM topograph. A constant height AFM scan indicated by the frame in Fig. 4A is shown in Fig. 4B. The energy spectrum of the 24 atom corral has only 10 states with 6 different eigenvalues $( \pm l$ states are degenerate) below $E_{\mathrm{F}}$ (Fig. $4 \mathrm{C}$ ). The average surface charge density of the 24 atom corral is similar to the 48 atom corral, but its magnitude $\sigma_{\text {total }}$ varies about three times as much, displayed in Fig. 4D. Correspondingly, the force contrast is about three times as high as for the 48 atom corral (Fig. 2E). To study the bonding properties of the small corral, we performed constant height AFM line scans over a height range from 450 to 1100 pm as shown in Fig. 4E, and the contrast is plotted as a function of distance over a height range from 450 to $550 \mathrm{pm}$ in Fig. 4F. The decay rate of the contrast with distance is at $l_{\mathrm{met}}{ }^{\prime}=50.5 \mathrm{pm}$ again near the calculated value of $l_{\mathrm{B}}=42.1 \mathrm{p} . \mathrm{m}$. [see section 12 in $(6)$ for more details].

STM has proven to be very useful for the study of quantized surface states (29-34), but it relies on tunneling electrons from the tip to the surface quantum state and to the bulk (or reverse), i.e., on transport. AFM not only allows to study the chemical bonding properties of these states, but also images stationary states that do not contribute to conduction [see section 13 in (6)].

\section{REFERENCES AND NOTES}

1. M. A. Kastner, Artificial atoms. Phys. Today 46, 24-31(1993). doi:10.1063/1.881393

2. M. F. Crommie, C. P. Lutz, D. M. Eigler, Confinement of electrons to quantum corrals on a metal surface. Science 262, 218-220 (1993). doi:10.1126/science.262.5131.218 Medline

3. D. M. Eigler, E. K. Schweizer, Positioning single atoms with a scanning tunnelling microscope. Nature 344, 524-526 (1990). doi:10.1038/344524a0

4. G. Binnig, C. F. Quate, C. Gerber, Atomic force microscope. Phys. Rev. Lett. 56, 930-933 (1986). doi:10.1103/PhysRevLett 56.930 Medline

5. S. Kawai, A. S. Foster, T. Björkman, S. Nowakowska, J. Björk, F. F. Canova, L. H. Gade, T. A. Jung, E. Meyer, Van der Waals interactions and the limits of isolated atom models at interfaces. Nat. Commun. 7, 11559 (2016). doi:10.1038/ncomms 11559 Medline

6. See the supplementary materials.

7. A. Zangwill, Physics at Surfaces (Cambridge Univ. Press, 1988).

8. P. O. Gartland, S. Berge, B. J. Slagsvold, Photoelectric work function of a copper single crystal for the (100), (110), (111), and (112) faces. Phys. Rev. Lett. 28, 738739 (1972). doi:10.1103/PhysRevLett.28.738

9. S. D. Kevan, Effective-mass theory of simple surface states. Phys. Rev. B Condens. Matter 34, 6713-6718 (1986). doi:10.1103/PhysRevB.34.6713 Medline

10. M. F. Crommie, C. P. Lutz, D. M. Eigler, Imaging standing waves in a twodimensional electron gas. Nature 363, 524-527 (1993). doi:10.1038/363524a0

11. E. J. Heller, M. F. Crommie, C. P. Lutz, D. M. Eigler, Scattering and absorption of surface electron waves in quantum corrals. Nature 369, 464-466 (1994). do: $10.1038 / 369464 a 0$

12. M. F. Crommie, C. P. Lutz, D. M. Eigler, E. J. Heller, Quantum corrals. Physica D 83, 98-108 (1995). doi:10.1016/0167-2789(94)00254-N

13. G. A. Fiete, E. J. Heller, Colloquium: Theory of quantum corrals and quantum mirages. Rev. Mod. Phys. 75, 933-948 (2003). doi:10.1103/RevModPhys.75.933

14. Here, we only count the 11 valence electrons of $C u$ as its core electrons have much more negative bonding energies, while the 102 corral electrons are all in a very narrow energy range from $-5.38 \mathrm{eV}$ to $-4.94 \mathrm{eV}$. We project the valence electrons onto the surface area of the atom to obtain a surface charge density rather than calculating a volume charge density. The reason is that the experimental situation of a 2D surface imposes a strong asymmetry between the in-plane coordinates $x$, y or $r$, and $z$, the coordinate normal to the surface.

15. T. R. Albrecht, P. Grütter, D. Horne, D. Rugar, Frequency modulation detection using high-Q cantilevers for enhanced force microscope sensitivity. J. Appl. Phys. 69, 668-673 (1991). doi:10.1063/1.347347

16. R. García, R. Perez, Dynamic atomic force microscopy methods. Surf. Sci. Rep. 47, 197-301 (2002). doi:10.1016/S0167-5729(02)00077-8

17. S. Morita, F. J. Giessibl, E. Meyer, R. Wiesendanger, Noncontact Atomic Force Microscopy (Springer, 2015), vol. 3.

18. M. Ternes, C. González, C. P. Lutz, P. Hapala, F. J. Giessibl, P. Jelínek, A. J. Heinrich, Interplay of conductance, force, and structural change in metallic point contacts. Phys. Rev. Lett. 106, 016802 (2011). doi:10.1103/PhysRevLett.106.016802 Medline

19. L. Bartels, G. Meyer, K.-H. Rieder, D. Velic, E. Knoesel, A. Hotzel, M. Wolf, G. Ertl, Dynamics of electron-induced manipulation of individual $\mathrm{CO}$ molecules on Cu(111). Phys. Rev. Lett. 80, 2004-2007 (1998). doi:10.1103/PhysRevLett.80.2004

20. L. Gross, F. Mohn, N. Moll, P. Liljeroth, G. Meyer, The chemical structure of a molecule resolved by atomic force microscopy. Science 325, 1110-1114 (2009). doi:10.1126/science. 1176210 Medline

21. N. Moll, L. Gross, F. Mohn, A. Curioni, G. Meyer, The mechanisms underlying the en- hanced resolution of atomic force microscopy with functionalized tips. New J. Phys. 12, 125020 (2010). doi:10.1088/1367-2630/12/12/125020

22. N. Moll, L. Gross, F. Mohn, A. Curioni, G. Meyer, A simple model of molecular imaging with noncontact atomic force microscopy. New J. Phys. 14, 083023 (2012). doi:10.1088/1367-2630/14/8/083023

23. B. Schuler, W. Liu, A. Tkatchenko, N. Moll, G. Meyer, A. Mistry, D. Fox, L. Gross, 
Adsorption geometry determination of single molecules by atomic force microscopy. Phys. Rev. Lett. 111, 106103 (2013). doi:10.1103/PhysRevLett.111.106103 Medline

24. M. Emmrich, F. Huber, F. Pielmeier, J. Welker, T. Hofmann, M. Schneiderbauer, D. Meuer, S. Polesya, S. Mankovsky, D. Ködderitzsch, H. Ebert, F. J. Giessibl, Surface structure. Subatomic resolution force microscopy reveals internal structure and adsorption sites of small iron clusters. Science 348, 308-311 (2015). doi:10.1126/science. aaa5329 Medline

25. J. Repp, F. Moresco, G. Meyer, K.-H. Rieder, P. Hyldgaard, M. Persson, Substrate mediated long-range oscillatory interaction between adatoms: $\mathrm{Cu} / \mathrm{Cu}(111)$. Phys. Rev. Lett. 85, 2981-2984 (2000). do:10.1103/PhysRevLett.85.2981 Medline

26. N. Knorr, H. Brune, M. Epple, A. Hirstein, M. A. Schneider, K. Kern, Long-range adsorbate interactions mediated by a two-dimensional electron gas. Phys. Rev. B Condens. Matter Mater. Phys. 65, 115420 (2002). doi:10.1103/PhysRevB.65.115420

27. K. Morgenstern, K.-F. Braun, K.-H. Rieder, Direct imaging of Cu dimer formation, motion, and interaction with Cu atoms on Ag(111). Phys. Rev. Lett. 93, 056102 (2004). doi:10.1103/PhysRevLett.93.056102 Medline

28. C. W. Groth, M. Wimmer, A. R. Akhmerov, X. Waintal, Kwant: A software package for quantum transport. New J. Phys. 16, 063065 (2014). doi:10.1088/13672630/16/6/063065

29. H. C. Manoharan, C. P. Lutz, D. M. Eigler, Quantum mirages formed by coherent projection of electronic structure. Nature 403, 512-515 (2000). doi:10.1038/35000508 Medline

30. K. K. Gomes, W. Mar, W. Ko, F. Guinea, H. C. Manoharan, Designer Dirac fermions and topological phases in molecular graphene. Nature 483, 306-310 (2012). doi:10.1038/nature10941 Medline

31. K. Seufert, W. Auwärter, F. J. García de Abajo, D. Ecija, S. Vijayaraghavan, S. Joshi, J. V. Barth, Controlled interaction of surface quantum-well electronic states. Nano Lett. 13, 6130-6135 (2013). doi:10.1021/nل403459m Medline

32. A. A. Khajetoorians, D. Wegner, A. F. Otte, I. Swart, Creating designer quantum states of matter atom-by-atom. Nat. Rev. Phys. 1, 703-715 (2019). doi:10.1038/s42254-019-0108-5

33. L. Yan, P. Liljeroth, Engineered electronic states in atomically precise artificial lattices and graphene nanoribbons. Adv. Phys. X 4, 1651672 (2019). doi:10.1080/23746149.2019.1651672

34. S. E. Freeney, S. T. P. Borman, J. W. Harteveld, I. Swart, Coupling quantum corrals to form artificial molecules. SciPost Phys. 9, 085 (2020). doi:10.21468/SciPostPhys.9.6.085

35. F. Queck-Scharrer, "Implementierung der Rastertunnelmikroskopie auf Isolatoren," PhD thesis, Physics Department, University of Regensburg, Regensburg, Germany (2019); https://epub.uni-regensburg.de/38271/

36. Numerical data for: F. Stilp, A. Bereczuk, J. Berwanger, N. Mundigl, K. Richter, F. J. Giessibl, Very weak bonds to artificial atoms formed by quantum corrals, University of Regensburg (2021); https://doi.org/10.5283/epub.45631.

37. F. J. Giessibl, The qPlus sensor, a powerful core for the atomic force microscope. Rev. Sci. Instrum. 90, 011101 (2019). doi:10.1063/1.5052264 Medline

38. J. Welker, F. J. Giessibl, Revealing the angular symmetry of chemical bonds by atomic force microscopy. Science 336, 444-449 (2012). doi:10.1126/science.1219850 Medline

39. F. Huber, F. J. Giessibl, Low noise current preamplifier for qPlus sensor deflection signal detection in atomic force microscopy at room and low temperatures. Rev. Sci. Instrum. 88, 073702 (2017). doi:10.1063/1.4993737 Medline

40. C. J. Chen, Introduction to Scanning Tunneling Microscopy (Oxford Univ. Press, 1993).

41. F. Huber, J. Berwanger, S. Polesya, S. Mankovsky, H. Ebert, F. J. Giessibl, Chemical bond formation showing a transition from physisorption to chemisorption. Science 366, 235-238 (2019). doi:10.1126/science.ayy3444 Medline

42. J. A. Stroscio, D. M. Eigler, Atomic and molecular manipulation with the scanning tunneling microscope. Science 254, 1319-1326 (1991). doi:10.1126/science. 254.5036.1319 Medline

43. M. Schneiderbauer, M. Emmrich, A. J. Weymouth, F. J. Giessibl, CO tip functionalization inverts atomic force microscopy contrast via short-range electrostatic forces. Phys. Rev. Lett. 112, 166102 (2014). doi:10.1103/PhysRevLett.112.166102 Medline
44. O. Gretz, A. J. Weymouth, F. J. Giessibl, Identifying the atomic configuration of the tip apex using STM and frequency-modulation AFM with CO on Pt(111). Phys. Rev. Res. 2, 033094 (2020). doi:10.1103/PhysRevResearch.2.033094

45. I. Horcas, R. Fernández, J. M. Gómez-Rodríguez, J. Colchero, J. Gómez-Herrero, A. M. Baro, WSXM: A software for scanning probe microscopy and a tool for nanotechnology. Rev. Sci. Instrum. 78, 013705 (2007). doi:10.1063/1.2432410 Medline

46. L. Gross, N. Moll, F. Mohn, A. Curioni, G. Meyer, F. Hanke, M. Persson, Highresolution molecular orbital imaging using a p-wave STM tip. Phys. Rev. Lett. 107, 086101 (2011). doi:10.1103/PhysRevLett.107.086101Medline

47. R. P. Feynman, R. B. Leighton, M. Sands, The Feynman Lectures on Physics II. (Addison-Wesley, 1963).

48. A. Liebig, A. Peronio, D. Meuer, A. J. Weymouth, F. J. Giessibl, High-precision atomic force microscopy with atomically-characterized tips. New J. Phys. 22, 063040 (2020). doi:10.1088/1367-2630/ab8efd

49. N. D. Lang, W. Kohn, Theory of metal surfaces: Charge density and surface energy. Phys. Rev. B 1, 4555-4568 (1970). doi:10.1103/PhysRevB.14555

50. F. J. Giessibl, A direct method to calculate tip-sample forces from frequency shifts in frequency-modulation atomic force microscopy. Appl. Phys. Lett. 78, 123-125 (2001). doi:10.1063/1.1335546

51. A. J. Weymouth, T. Wutscher, J. Welker, T. Hofmann, F. J. Giessibl, Phantom force induced by tunneling current: A characterization on Si(111). Phys. Rev. Lett. 106, 226801 (2011). doi:10.1103/PhysRevLett.106.226801 Medline

\section{ACKNOWLEDGMENTS}

We thank Christopher P. Lutz for help with creating the original type of a $48 \mathrm{Fe}$ atom corral on $\mathrm{Cu}(111)$. FJG is grateful to Andreas Heinrich and Christopher P. Lutz for hosting several research visits at the IBM Almaden Research Center from 2005 until 2010. We further thank Jascha Repp and his former PhD student Fabian Queck, whose study of linear quantum resonators by combined STM and AFM [chapter 3 in (35)] has contributed to our interest in looking at possible force contrast in the quantum corral. We are very grateful to Gerd Binnig. Michael Crommie, Donald Eigler, Gerhard Ertl, James Gimzewski, Roald Hoffmann, Christopher Lutz, Marlou Slot and Joseph Stroscio for suggesting many improvements of the manuscript. We also thank Alexander Liebig, Marco Weiss, and Jay Weymouth for discussions and proof reading, Michael Barth for advice in the numerical simulations and Anja Merkel for creating the model of the corral shown in figure S2. Funding: We thank the Deutsche Forschungsgemeinschaft (DFG, German Research Foundation) for funding under Project-ID 314695032 SFB 1277 projects A02 (FS, JB, NM, FJG) and A07 (AB, KR). Author contributions: Conceptualization: FJG, KR; Methodology: FS, AB, KR, JB, NM, FJG; Investigation: FS, AB, JB, NM; Visualization: FS, AB, FJG; Funding acquisition: FJG, KR; Project administration: FJG, KR; Supervision: FJG, KR, JB; Writing - original draft: FJG; Writing - review and editing: FS, AB, KR, JB, FJG. Competing interests: FJG holds patents about the force sensor that was used in the experiments. All other authors declare no competing interests. Data and materials availability: All experimental data are available in the main text or the supplementary materials, numerical data are available at (36).

\section{SUPPLEMENTARY MATERIALS}

science. sciencemag.org/cgi/content/full/science.abe2600/DC1

Materials and Methods

Figs. S1 to S22

Tables S1 to S3

References (37-51)

10 August 2020; resubmitted 10 March 2021

Accepted 3 May 2021

Published online 13 May 2021

$10.1126 /$ science.abe2600 

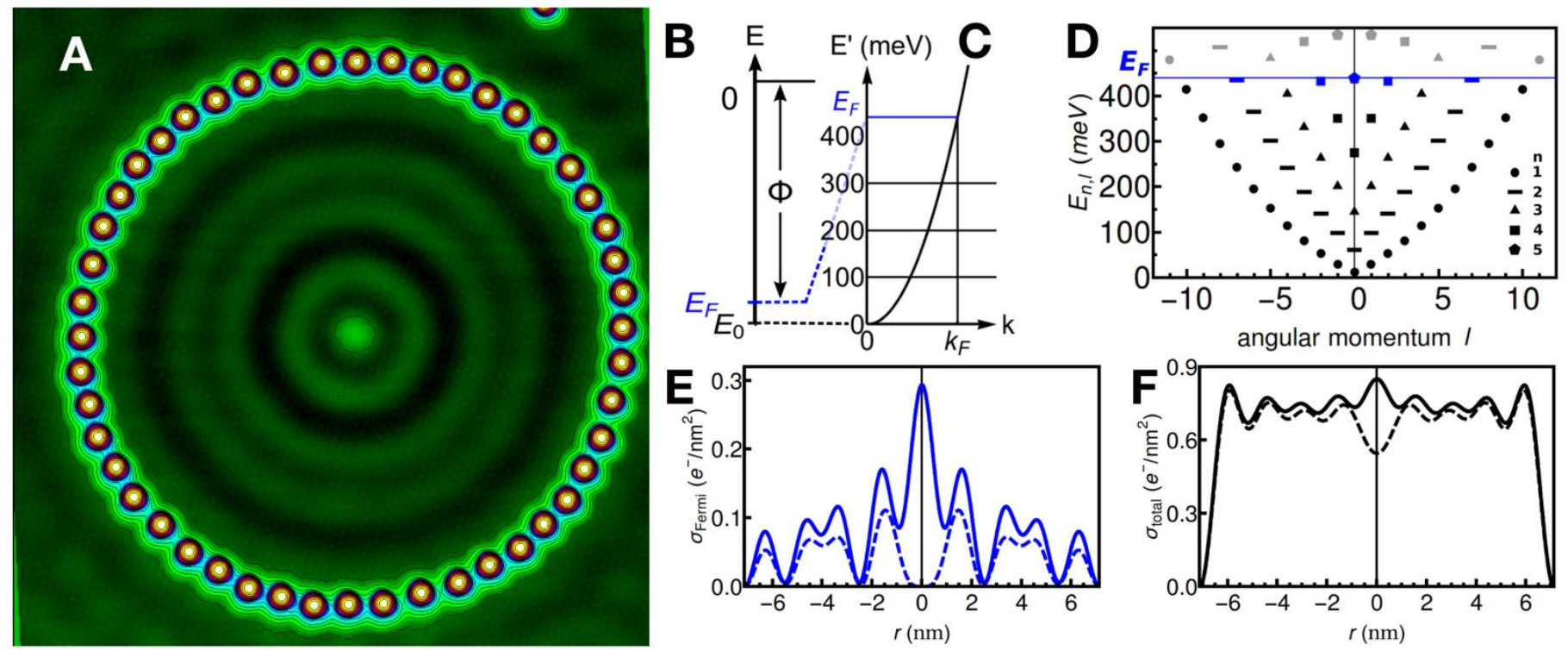

Fig. 1. Quantum corral consisting of $48 \mathrm{Fe}$ atoms on $\mathrm{Cu}$ (111). (A) Topographic STM image at a tip bias of -1 $\mathrm{mV}$ and a tunneling current of $20 \mathrm{pA}$ using a CO terminated tip. The ring has a radius of $7.13 \mathrm{~nm}$, the Cu atoms of the $\mathrm{Cu}(111)$ substrate appear as peaks with a height of $\approx 500 \mathrm{fm}$ and a distance of 255 p.m.(B) Energy diagram of the electrons on the $\mathrm{Cu}(111)$ surface with vacuum level at $\mathrm{O}$, the Fermi energy $E_{\mathrm{F}}$ at $-4.94 \mathrm{eV}$, and the lowest energy of the surface state $E_{0}$ another $-0.44 \mathrm{eV}$ lower. (C) Energy spectrum of the occupied surface states on the Cu(111) surface with a shifted energy scale $E^{\prime}=E-E_{0}$ and dispersion relation $E^{\prime}=\hbar^{2} k^{2} /\left(2 m^{*}\right)$. (D) Energy spectrum of the occupied corral states, characterized by the number of zeroes $n$ and the angular momentum I. The states at the Fermi level (blue) are imaged by STM at low bias (2). (E) Calculated surface charge density $\sigma_{\text {Fermi }}$ of the states at the Fermi level, dashed line without contribution of $\psi_{5,0}$. (F) Calculated surface charge density of all occupied states $\sigma_{\text {total }}$, dashed line without contribution of $\psi 5,0$. Surface charge densities are calculated using the Bessel-type eigenstates defined in (6). 


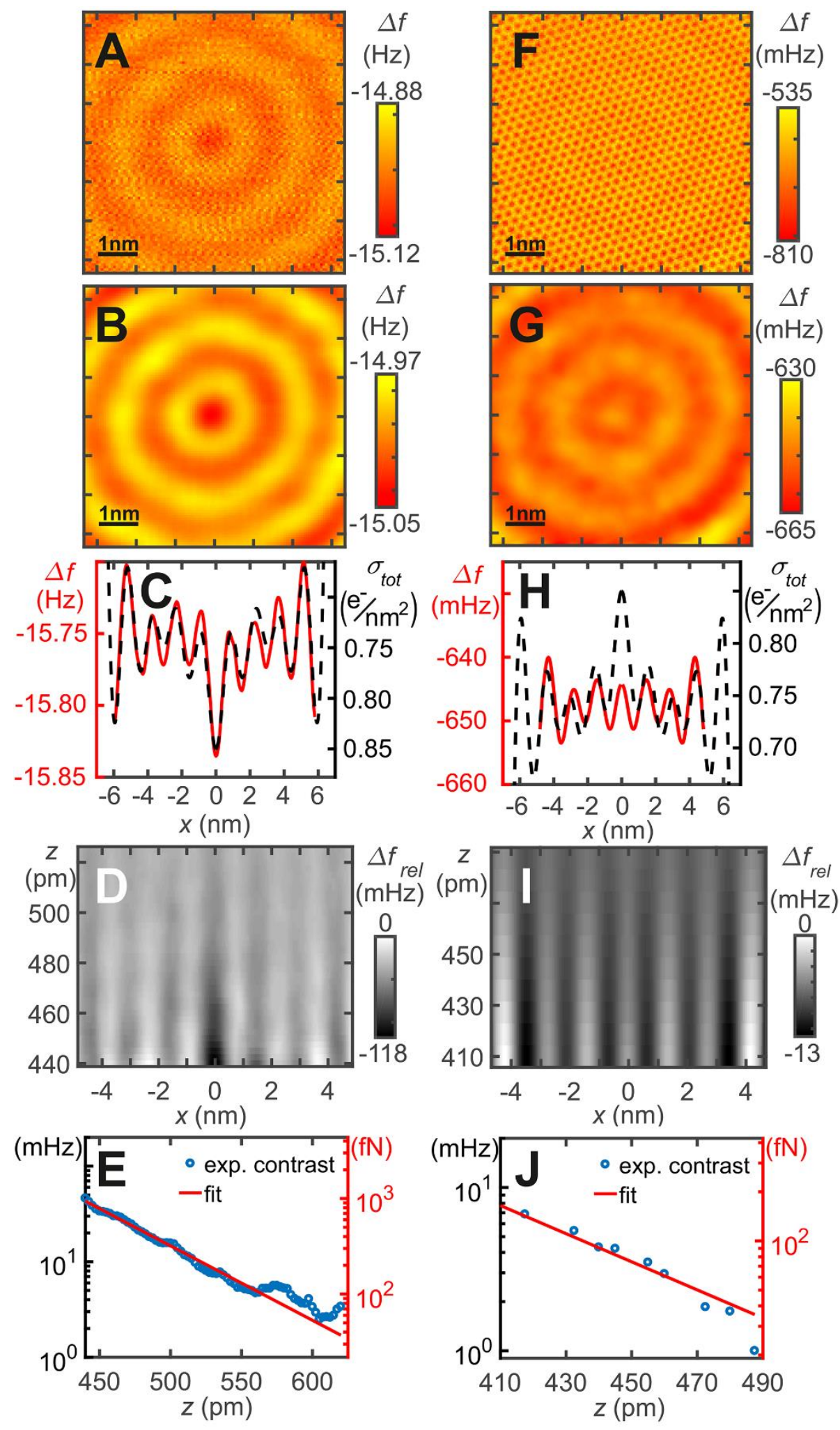

Fig. 2. AFM data inside the quantum corral for a single atom metal tip (left column) and a CO terminated tip (right column). (A) Frequency shift data $\Delta f(x, y, z=$ const.) with an attractive interaction between the probe tip and the Bessel-type eigenstates. (B) Low-pass filtered version of (A). (C) Profile line through the center of $B$ and reversed total surface charge density of the corral. (D) Gray-scale representation of offset and slope corrected frequency shift $\Delta f_{\mathrm{rel}}(x, 0, z)$. (E) Distance dependence of the contrast, defined as the difference between the average of the first and second maxima and the minimum in between. (F) Frequency shift data $\Delta f(x, y, z=$ const.), showing attraction to the Cu surface atoms and repulsion to the Bessel-type eigenstates. (G) Low- pass filtered version of $F$, showing only the repulsion to the Bessel-type eigenstates. (H) Profile line through center of $\mathrm{G}$ compared to total charge density. (I) Gray scale presentation of offset and slope corrected frequency shift $\Delta f_{\mathrm{rel}}(x, 0, z)$. (J) Contrast, defined by the difference between the first maximum at $x \approx \pm 1.3 \mathrm{~nm}$ and the average between the first and second minimum, evolution as a function of vertical distance $z$. See section 7 in (6) for details of data extraction. 


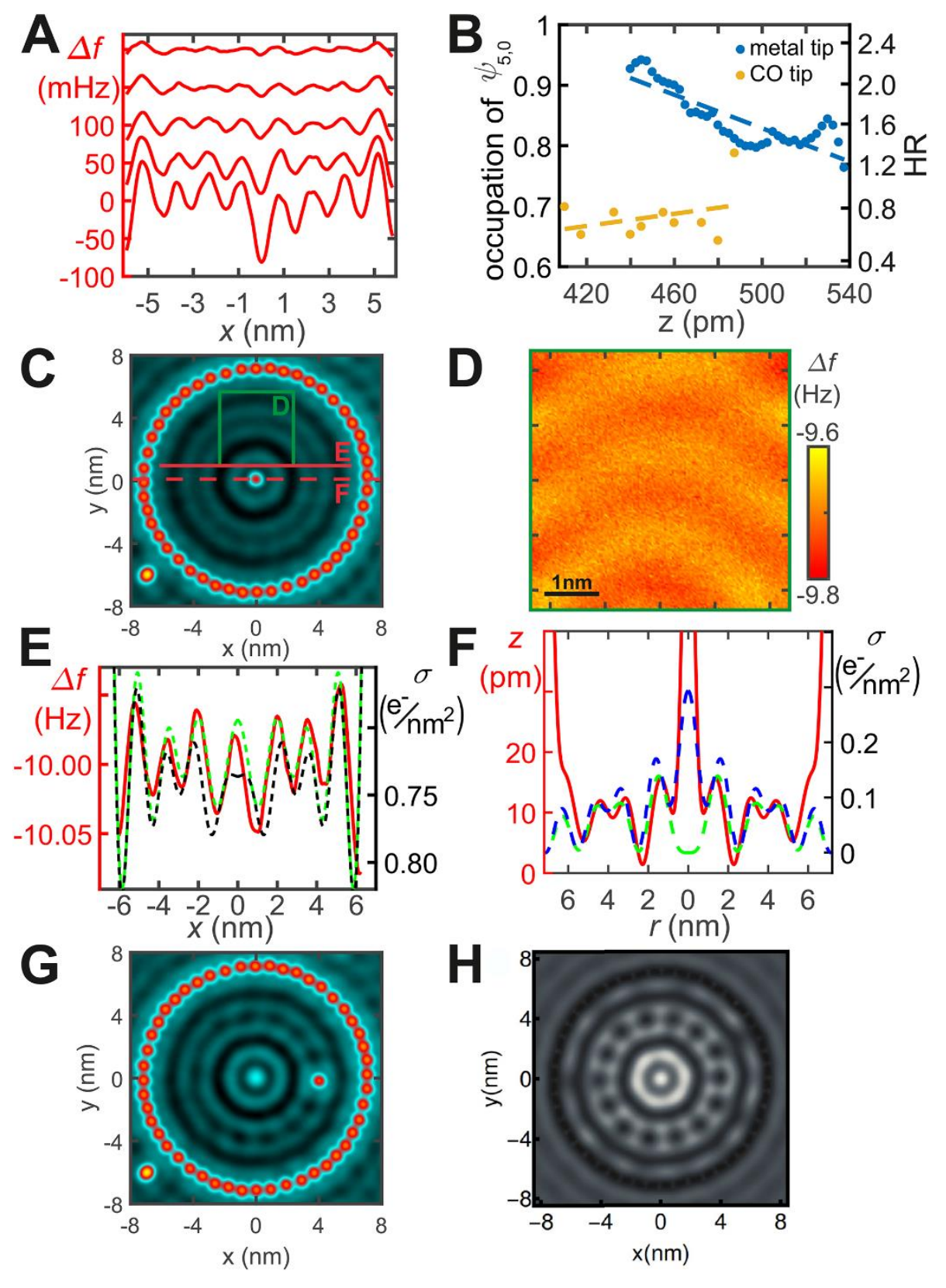

Fig. 3. Impact of the perturbation inflicted by the AFM tip $[(A)$ and $(B)]$ and a deliberately placed metal adatom inside $[(\mathrm{C})$ to $(\mathrm{H})]$. (A) AFM traces (single atom metal tip) through the center of the corral for five different heights. (B) Relative depth of the central dip in traces from A as a function of distance $z$ and corresponding occupation of $\psi_{5,0}$ with a metal tip (blue) and a CO terminated tip (yellow). (C) Topographic STM image of the corral with an extra Fe adatom located at the center of the corral. Tip bias $-10 \mathrm{mV}$, current $10 \mathrm{pA}$ (D) Constant height AFM data within green square in (C). (E) Off-center line trace of $\Delta f(x, y=830$ p.m., $z=$ const.), shown as red trace in (C) and comparison to total charge density $\sigma_{\text {total }}$ (black) and $\sigma_{\text {total }}$ minus the contribution of $\psi_{5,0}$ (green). (F) Central STM topography trace (red) through the corral with an additional adatom in its center and comparison to full contribution of $\psi_{5,0}, \psi_{4, \pm 2}$, and $\psi_{2, \pm 7}$ (blue) states and without the $\psi_{5,0}$ state (green). (G) Experimental topographic STM image of the corral with an extra Fe adatom located at $r=4.048 \mathrm{~nm}$ and $\phi=0$. The states $\psi_{2, \pm 7}$ have a local maximum close to $r=4 \mathrm{~nm}$, therefore the $\cos ^{2}(7 \phi)$ component is lifted in energy and its occupation is reduced, while its $\sin ^{2}(7 \phi)$ component is affected less. Tip bias $-10 \mathrm{mV}$, current $10 \mathrm{pA}$. (H) Calculated topographic STM image of G using the tight binding simulations (6). Note that the direct current channel into the corral atoms and extra Fe atom is not considered in the calculation, similar as in Fig. 3 of (11). 

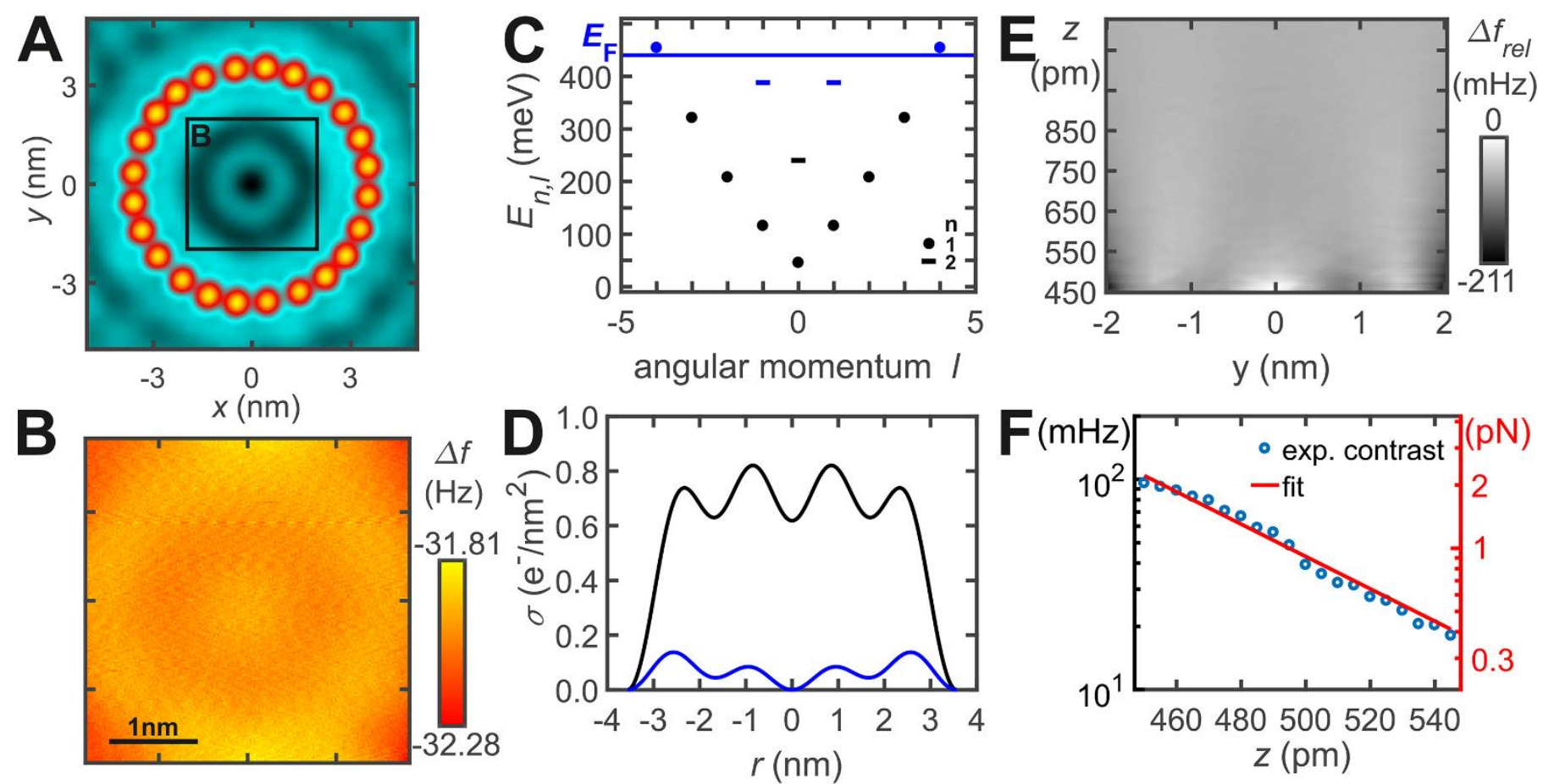

Fig. 4. Quantum corral with $24 \mathrm{Fe}$ adatoms and a radius of $r_{\mathrm{C}^{\prime}}=3.565 \mathrm{~nm}$. (A) STM topograph at tip bias $-10 \mathrm{mV}$. (B) Constant height AFM data inside the corral. (C) Energy spectrum, states near $E_{F}$ in blue. (D) Total charge density Stotal' (black) modeled by using a weight of 1 for all occupied states and charge density at the Fermi level SFermi' (blue) modeled by a weight of 1 for the $y_{1, \pm}$ states and a weight of 0.4438 for the $y_{2, \pm 1}$ states [see section 12 of (6) for details]. (E) Frequency shift as a function of lateral and vertical coordinates $y$ and $z$. (F) Plot of contrast in frequency shift as a function of vertical coordinate $z$ showing an exponential decay with a decay length $I_{\text {met }}{ }^{\prime}=50.5$ p.m. 